\title{
Atypical Presentation of Esophageal Squamous Cell Carcinoma Masquerading as Diffuse Severe Esophagitis
}

\author{
Hafsa Abbas ${ }^{a}$ Haider Ghazanfar ${ }^{b}$ Ali Naqqi UI Hussain ${ }^{c}$ \\ Ahmed Baiomi $^{a}$ Ariyo Ihimoyan ${ }^{a}$ \\ aDepartment of Gastroenterology, Bronxcare Health System, Bronx, NY, USA; ${ }^{b}$ Department \\ of Medicine, Bronxcare Health System, Bronx, NY, USA; 'Baruch College, City University of \\ New York (CUNY), New York, NY, USA
}

\author{
Keywords \\ Esophagitis - Dysphagia - Gastroesophageal reflex disease - Squamous cell carcinoma of the \\ esophagus
}

\begin{abstract}
Esophagitis is described as an inflammation of the esophagus and can result from multiple etiologies. Esophageal squamous cell carcinoma (ESCC), presenting as diffuse esophagitis in the absence of a mass or lesion, is rare. We present a case of a 61-year-old man who presented to the gastroenterology clinic for dysphagia and heartburn of 3 months duration. The patient had lost about 15 pounds unintentionally over 6 months. The patient underwent esophagogastroduodenoscopy, which revealed significant diffuse Grade 4 esophagitis without any overt bleeding. Random biopsies were taken with cold forceps from proximal, middle, and distal esophageal segments because of the striking endoscopic appearance. Histopathology revealed high-grade dysplasia and carcinoma in situ. The patient underwent endoscopic ultrasound (EUS) of the esophagus, which revealed a focal lesion. EUS-guided fine-needle aspiration showed squamous cell carcinoma of the esophagus. ESCC usually presents as a mass or a gross lesion seen on endoscopy. However, it rarely presents as severe diffuse esophagitis seen on routine endoscopy. From our observation, it would be reasonable for physicians to bear this unusual endoscopic presentation in mind and perform multiple random biopsies if encountered with such a case to rule out the possibility of any underlying malignancy.
\end{abstract}

\section{Karger ${ }^{\prime \prime}=$}




\section{Introduction}

Esophagitis is a well-known condition described as an inflammation of the esophagus. There are various etiologies of esophagitis, including infectious, noninfectious, chemical, medication-induced, and gastroesophageal reflux disease. These etiologies commonly involve the distal esophagus. The history and physical findings may vary according to the underlying etiology. The most common symptoms of esophagitis include heartburn, dyspepsia, nausea, bloating, and fullness. Less common clinical features of esophagitis include dysphagia and odynophagia. According to a study in the USA, among patients with symptoms of gastroesophageal reflux, around 34\% were found to have reflux esophagitis on esophagogastroduodenoscopy (EGD) [1]. Savary-Miller is the most widely used endoscopic grading system used to grade the degree of esophagitis. Common complications of GERD include ulceration, stricture formation, and Barrett esophagus, which can eventually progress to esophageal adenocarcinoma.

Esophageal cancer is associated with a high mortality and is the eighth most common cause of cancer in the world [2]. According to statistical analysis done by the American Cancer Society 16, 170 deaths are expected to result from esophageal cancer [3]. Poor nutritional status, low intake of vegetables and fruit, and consumption of hot beverages are thought to contribute to squamous cell carcinoma of the esophagus in the developing countries, while smoking and excessive alcohol consumptions are thought to contribute to the development of $90 \%$ of total cases of squamous cell carcinoma of the esophagus in the USA and several Western countries [4]. According to a study done in 22 centers in North America, Europe, and Australia, it the incidence of adenocarcinoma of the esophagus has increased significantly over the last 20 years [5]. The same study revealed that the average increase in the incidence has been from 8.7 to $17.5 \%$.

Esophageal squamous cell carcinoma (ESCC), presenting as diffuse esophagitis in the absence of a mass or lesion, is rare. We present a case of severe diffuse esophagitis in a patient with grade 4 esophagitis and which was later found to be ESCC on the pathology of the routine biopsies.

\section{Case Presentation}

We present a case of 61-year-old man who presented to the outpatient gastroenterology clinic for dysphagia of 3 months duration. He complained of solid food getting stuck in his chest and had mild discomfort with liquids as well. He also reported some heartburn and acid reflux which had not responded to over-the-counter antacids and ranitidine. He reported unintentional weight loss from 200 pounds to 185 pounds over 6 months. There was no abdominal pain, nausea, vomiting, constipation, fever, and early satiety or appetite changes, hematemesis, hematochezia, or melena. He had a prior history of hypertension, hyperlipidemia. He had an inguinal hernia repair and left knee surgery in the past. He also had a screening colonoscopy a few years prior to his presentation and was unremarkable except right-sided diverticulosis and nonbleeding internal hemorrhoids.

He was a current smoker and admitted smoking cigarettes everyday but denied using alcohol except on social occasions or illicit drugs. He did not have a family history of liver, stomach, or colon cancer. His medications included aspirin, atorvastatin, carvedilol, and amlodipine.

On examination, he was afebrile, and his vital signs were within normal limits. He was alert, fully oriented, and communicative. The abdomen was soft, nontender, nondistended with normal bowel sounds. Spleen was palpable on physical examination. Cervical and supraclavicular lymph nodes were not palpable. Rest of the physical examination was unremarkable. Patient complete blood cell count, basic metabolic profile, and liver function tests were ordered. They were unremarkable.

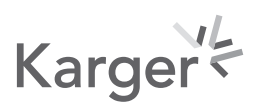


Fig. 1. Endoscopic photograph showing grade 4 esophagitis.

Fig. 2. Histopathology of the distal esophagus showing high-grade dysplasia and carcinoma in situ (original magnification is $\times 100$ ).
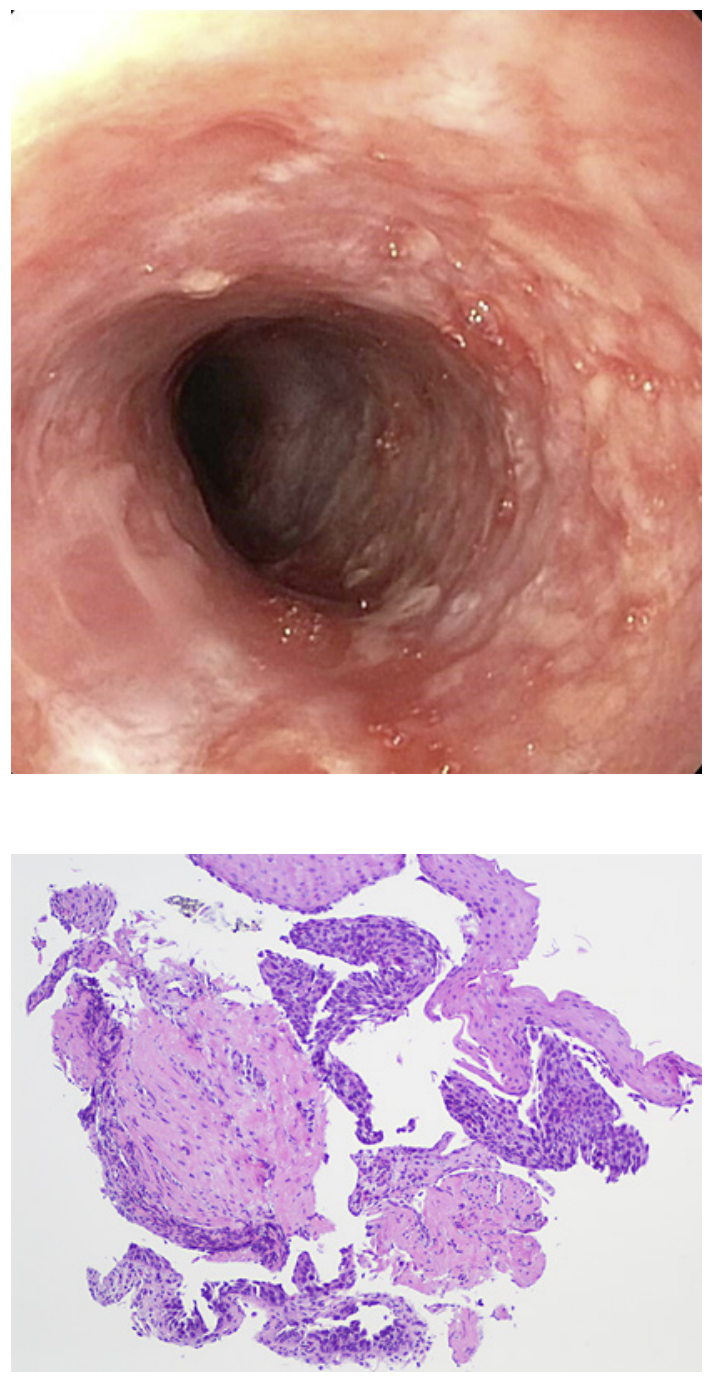

Patient underwent EGD which revealed significant Grade 4 esophagitis in the entire esophagus. This has been shown in Figure 1.

Random biopsies were taken with a cold forceps from 3 different segments of the esophagus because of the high-risk clinical feature of the patient and were sent for further analysis. Sample was also sent for Helicobacter pylori testing. Histopathology revealed highgrade dysplasia and carcinoma in situ. This has been shown in Figures 2 and 3.

Patient was referred to a tertiary care hospital center for further management. Patient underwent endoscopic ultrasound of esophagus which revealed a focal lesion. Endoscopic ultrasound-guided fine-needle aspiration showed ESCC. Oncology was consulted for further management.

\section{Discussion}

Various factors contribute to the development of ESCC. Risk factors for the development of ESCC include hereditary factors, smoking and alcohol consumptions, high-temperature beverages, a diet low in vegetables and fruits, preexisting esophageal diseases such as 
Fig. 3. Magnified histopathology of the distal esophagus showing high-grade dysplasia and carcinoma in situ (original magnification is $\times 400$ ).

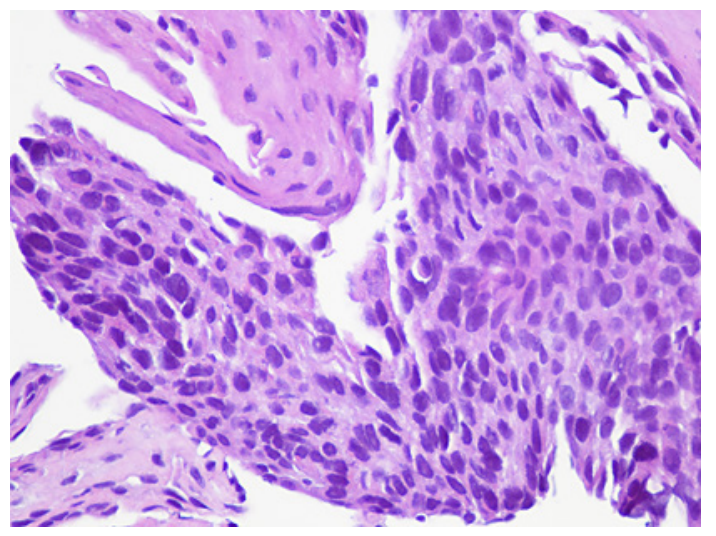

achalasia and caustic strictures, prior partial gastrectomy, atrophic gastritis, and human papillomavirus [6-9]. The prevalence of ESCC increases with age and peaks around seventh decade of life [10]. The incidence of esophageal SCC is higher in the African-American population and is more common in males than females [11].

Dysphagia and weight loss are the most common presenting symptoms of squamous cell carcinoma. The patient may also present with epigastric or retrosternal pain, regurgitation, persistent cough, hoarseness, and chronic gastrointestinal blood loss. Physical examination of the patient will be unremarkable unless esophageal cancer has metastasized. Our patient was a 61-year-old male who was an active smoker, presented with the complaint of dysphagia, heartburn, and weight loss.

Endoscopy with biopsy is the primary diagnostic tool. Endoscopic findings of early ESCC include irregular mucosa focal red area, small white patch, erosion, superficial plaques, nodules, or ulceration. The advanced lesion usually appears as stricture, ulceration, and large mucosal mass. White light endoscopy has a $62 \%$ sensitivity and specificity of $79 \%$ in detecting high-grade dysplasia and squamous cell cancer [12]. Our patient only had inflammation as the gross finding on EGD. Multiple random biopsies should be performed in patients with high-risk clinical features, risk factors, and endoscopic findings of severe diffuse esophagitis.

Lugol chromoendoscopy is a procedure in which Lugol's iodine is instilled into the gastrointestinal tract at the time of endoscopy. According to a study, the detection rate of esophageal dysplasia and early ESCC was $13.7 \%$ in the group of patients who underwent chromoendoscopy compared to $10.6 \%$ in the group of patients who underwent routine endoscopy ( $p=0.003$ ) [13]. We did not perform lugol chromoendoscopy for our patient.

A multidisciplinary approach is needed to manage patients with ESCC. According to Japan Esophageal Society (JES), endoscopic therapy can be considered in patients with mucosal tumors involving the epithelium and lamina propria mucosae. Surgical management can be considered in patient with localized disease. Preoperative chemoradiation therapy can be offered to patients with advanced noncervical esophageal cancer [14].

Despite advances in treatment modalities for esophageal cancer, the overall 5-year survival rate of patients with esophageal cancer is still 15-25\% worldwide [15]. Tumor size, tumor staging, node staging, and tumor differentiation are independent prognostic factors affecting the prognosis of ESCC patients [16].

Squamous cell carcinoma usually presents as a mass or a lesion seen on endoscopy. However, it rarely presents as severe diffuse esophagitis found on routine endoscopy. From our observation, we conclude it would be reasonable for physicians to bear this unusual endoscopic presentation in mind and perform multiple random biopsies if encountered with such 
a case to rule out the possibility any an underlying malignancy which if missed could have life-threatening consequences.

\section{Statement of Ethics}

Written informed consent was obtained from the patient for the publication of this case report. This study protocol was reviewed and the need for approval was waived by Bronxcare Health System Ethics Committee.

\section{Conflict of Interest Statement}

The author(s) of this manuscript do(es) not have any conflicts of interest to declare.

\section{Funding Sources}

There was no external source of funding to support this case report.

\section{Author Contributions}

Each of the authors contributed equally in conceiving the idea for the case and the research and helped to draft the manuscript and the image. All the authors read and approved the final manuscript.

\section{References}

1 Wo JM, Mendez C, Harrell S, Joubran R, Bressoud PF, McKinney WP. Clinical impact of upper endoscopy in the management of patients with gastroesophageal reflux disease. Am J Gastroenterol. 2004;99(12):2311-6.

2 Napier KJ, Scheerer M, Misra S. Esophageal cancer: a review of epidemiology, pathogenesis, staging workup and treatment modalities. World J Gastrointest Oncol. 2014;6(5):112-20.

3 Siegel RL, Miller KD, Jemal A. Cancer statistics, 2020. CA Cancer J Clin. 2020;70(1):7-30.

4 Engel LS, Chow WH, Vaughan TL, Gammon MD, Risch HA, Stanford JL, et al. Population attributable risks of esophageal and gastric cancers. J Natl Cancer Inst. 2003 Sep 17;95(18):1404-13.

5 Bollschweiler E, Wolfgarten E, Gutschow C, Hölscher AH. Demographic variations in the rising incidence of esophageal adenocarcinoma in white males. Cancer. 2001 Aug 1;92(3):549-55.

6 Pandeya N, Williams G, Green AC, Webb PM, Whiteman DC. Australian Cancer Study. Alcohol consumption and the risks of adenocarcinoma and squamous cell carcinoma of the esophagus. Gastroenterology. 2009 Apr; 136(4):1215-24, e1-2.

7 Islami F, Boffetta P, Ren JS, Pedoeim L, Khatib D, Kamangar F. High-temperature beverages and foods and esophageal cancer risk: a systematic review. Int J Cancer. 2009;125(3):491-524.

8 Islami F, Sheikhattari P, Ren JS, Kamangar F. Gastric atrophy and risk of oesophageal cancer and gastric cardia adenocarcinoma: a systematic review and meta-analysis. Ann Oncol. 2011;22(4):754-60.

9 Li X, Gao C, Yang Y, Zhou F, Li M, Jin Q, et al. Systematic review with meta-analysis: the association between human papillomavirus infection and oesophageal cancer. Aliment Pharmacol Ther. 2014 Feb;39(3):27081.

10 Zhang Y Epidemiology of esophageal cancer. World J Gastroenterol. 2013;19(34):5598-606.

11 Layke JC, Lopez PP. Esophageal cancer: a review and update. Am Fam Physician. 2006;73(12):2187-94.

12 Domper Arnal MJ, Ferrández Arenas Á, Lanas Arbeloa Á Esophageal cancer: risk factors, screening and endoscopic treatment in Western and Eastern countries. World J Gastroenterol. 2015;21(26):7933-43.

13 Shao Y, Yu ZL, Ji M, Wu YD, Yu YZ, Liang XM, et al. Lugol chromoendoscopic screening for esophageal dysplasia/ early squamous cell carcinoma in patients with esophageal symptoms in low-risk region in China. Oncol Lett. 2015 Jul;10(1):45-50. 
14 Wong I, Law S. The management of mid \& proximal oesophageal squamous cell carcinoma. Best Pract Res Clin Gastroenterol. 2018 0ct-Dec;36-37:85-90.

15 Pennathur A, Gibson MK, Jobe BA, Luketich JD. Oesophageal carcinoma. Lancet. 2013 Feb 2;381(9864):40012.

16 Zhong H, Ma R, Gong L, Chen CG, Tang P, Ren P, et al. [Comparison of the prognostic value of the seventh and eighth edition of The AJCC Esophageal Cancer Staging System for the patients with stage II and III esophageal squamous cell carcinoma]. Zhonghua Wai Ke Za Zhi. 2017 Dec 1;55(12):903-8. Chinese. 